\title{
Photoionization of KCs Molecule: Origin of the Structured Continuum?
}

\author{
Goran Pichler ${ }^{1, *}$, Robert Beuc ${ }^{1}$, Jahja Kokaj ${ }^{2}$, David Sarkisyan ${ }^{3}$, Nimmy Jose ${ }^{2}$ \\ and Joseph Mathew ${ }^{2}$ \\ 1 Institute of Physics, 10000 Zagreb, Croatia; beuc@ifs.hr \\ 2 Department of Physics, Kuwait University, P.O. Box 5969, Safat 13060, Kuwait; jkokaj@yahoo.com (J.K.); \\ nimmyjosev@gmail.com (N.J.); mathewaerath@yahoo.com (J.M.) \\ 3 Institute for Physical Research, Armenian Academy of Science, Ashtarak 0203, Armenia; \\ davsark@yahoo.com \\ * Correspondence: pichler@ifs.hr; Tel.: +385-91-469-8826
}

Received: 30 April 2020; Accepted: 26 May 2020; Published: 28 May 2020

check for updates

\begin{abstract}
We report the experimental observation of photoionization bands of the KCs molecule in the deep ultraviolet spectral region between 200 and $420 \mathrm{~nm}$. We discuss the origin of observed photoionization bands as stemming from the absorption from the ground state of the KCs molecule to the excited states of $\mathrm{KCs}^{+}$molecule for which we used existing potential curves of the $\mathrm{KCs}^{+}$molecule. An alternative explanation relies on the absorption from the ground state of the KCs molecule to the doubly excited states of the KCs ${ }^{* *}$ molecule, situated above the lowest molecular state of $\mathrm{KCs}^{+}$. The relevant potential curves of $\mathrm{KCs}^{* *}$ are not known yet, but all those $\mathrm{KCs}^{* *}$ potential curves are certainly autoionizing. However, these two photoionization pathways may interfere resulting in a special interference structured continuum, which is observed as complex bands.
\end{abstract}

Keywords: photoionization; alkali molecule; autoionization of molecule; heteronuclear molecule

\section{Introduction}

High-temperature alkali mixtures possessing high densities of constituents enable observation of the characteristic bands of mixed alkali molecules [1]. Heavy alkali heteronuclear molecules are interesting because they have a permanent dipole moment which enables many applications like orientation in an external electric field and quantum computing. Additionally, spectral transitions are relaxed because there are no gerade and ungerade symmetries in their quantum state description, as is the case in homonuclear dimers. As a polar molecule, the KCs molecule, with a permanent dipole moment of 1.92 Debye, has been extensively studied by several research groups using high-resolution spectroscopy techniques. The lower excited states with asymptotes at K(4s) $+\mathrm{Cs}(6 \mathrm{p})$ and/or $\mathrm{K}(4 \mathrm{p})+\mathrm{Cs}(6 \mathrm{~s})$ were usually the goals of several groups [2,3]. Some of the KCs intermediate high excited states were also investigated [4,5], of which the E state has a shelf-like shape. Even the formation of ultracold polar ground state KCs molecules via an optical process has been discussed in [6]. Much higher excited states and, especially, Rydberg molecular states have been much less studied.

The structured photoionization continuum was first observed by Marr and Creek [7] with the conclusion that molecular photoionization is behind the process. Later, Hotop and Niemax [8-10] interpreted observed bands as atomic-pair absorption bands. The final resolution of the atomic or molecular nature of the problem of the observed structured bands within the photoionization continuum came from the time of flight (TOF) measurements by Suemitsu and Samson [11]. Recently, a structured photoionization continuum was studied by Pichler et al. [12], where the superheating of cesium vapor was used to reveal the molecular origin of the observed absorption bands. Quite recently, 
a structured photoionization continuum of $\mathrm{Rb}_{2}$ was measured at very high temperatures of up to $600{ }^{\circ} \mathrm{C}$, which enabled the appreciable enhancement of the sought absorption bands [13]. This measurement confirmed the molecular origin of the observed rubidium absorption photoionization bands.

There are autoionizing [14] molecular doubly excited states (DES) above the ground molecular ion, such as $\mathrm{Cs}_{2}{ }^{* *}$ and $\mathrm{Rb}_{2}{ }^{* *}$ dimers, which have not yet been well investigated and are not fully supported by adequate quantum-mechanical calculations. The RbCs molecule in the visible spectrum has already been studied at high temperatures and densities in an all-sapphire cell [15] and the interpretation of some of the observed bands used existing potential curves [16].

In the present work, we undertook to study the structured photoionization continuum which appeared in the form of the absorption bands of the KCs molecule in the 220,300 nm spectral interval. We adopt the conclusion of the previous works [7,11-13] that observed photoionization KCs bands are of molecular origin. For such a study, it was very important to use an all-sapphire cell which enabled measurements of absorption coefficient at very high temperatures and particle densities. Much of the observed visible spectra were previously interpreted with ab initio quantum-mechanical calculations using potential energy curves calculated by the Allouche and Korek group [17]. In the present study, we were mainly interested in searching for the autoionizing molecular states of KCs lying above the ground state of the molecular ion $\mathrm{KCs}^{+}$. Some of the related photoionization bands were initially studied by Hotop and Niemax almost 40 years ago in 1981, using a very sensitive thermionic diode filled with equal quantities of potassium and cesium. Unfortunately, some of the photoionizing $\mathrm{KCs}$ bands were hidden by $\mathrm{Cs}_{2}$ photoionizing bands [10]. We, therefore, used the mixture of $\mathrm{K}[80 \%]$ and of Cs [20\%] which reduced $\mathrm{Cs}_{2}$ absorption and enabled clear observation of eight KCs photoionization bands in the spectral region 220-300 nm without disturbance from the presence of either $\mathrm{Cs}_{2}$ or $\mathrm{K}_{2}$ photoionization bands. We interpreted these bands as $\mathrm{KC}$ ground state transitions to (a) to excited states of the $\mathrm{KCs}^{+}$molecule [18] or (b) autoionizing doubly excited molecular states (DES) of a neutral KCs molecule lying above the first ionization limit (lowest molecular state of the $\mathrm{KCs}^{+}$ion). We could not run the experiment to very high temperatures and densities because the presence of hot potassium atoms in the alkali mixture would destroy the special glue at the sapphire windows. We, therefore, measured those photoionization bands up to $600^{\circ} \mathrm{C}$, which was well above the suggested secure limit of about $450^{\circ} \mathrm{C}$. Higher temperatures and densities of $\mathrm{KC}$ would be possible using a heat-pipe oven in which the windows are not in direct contact with aggressive alkali vapor.

\section{Experimental Background}

In our absorption experiment, the background lamp was a Deuterium lamp (Hamamatsu) and the spectrometer was OceanOptics USB4000 ES with enhanced sensitivity in the UV spectral region. A T-type all-sapphire cell (ASC), $4 \mathrm{~cm}$ in length and $1 \mathrm{~cm}$ in inner diameter, was used for the transmission measurements in the K-Cs mixture vapor. The concentration of these components could be determined approximately by the temperature at the tip of the side-arm finger $\left(T_{f}\right)$ and the cell body $\left(T_{c}\right)$. $T_{C}$ was always kept higher than $\mathrm{T}_{\mathrm{f}}$ by at least $30^{\circ} \mathrm{C}$ to prevent condensation of the vapors on the inside surface of both sapphire windows. The optical axes of the cell windows were perpendicular to the plane of the windows to avoid birefringence. A special glass extension, a few $\mathrm{mm}$ in length, was attached to the sapphire side-arm to enable filling with the mixture. The temperature of the cell body should not usually exceed $450{ }^{\circ} \mathrm{C}$. However, we managed up to $\mathrm{T}_{\mathrm{f}}=600^{\circ} \mathrm{C}$ after the subsequent four weeks of daily measurements by slowly increasing the cell temperature without damaging the cell. All previous ASC cells were filled either with pure potassium or with K-Cs mixtures which could easily be damaged because of the aggressive action of hot potassium atoms on the special glue used in the construction of the ASC. The oven, in which the quartz tubes were wound round by nichrome wire, consisted of two parts. The temperature of the two parts of the oven was controlled by two Chromel-Alumel Thermocouples (CAT). A third CAT measured the temperature $\left(\mathrm{T}_{\mathrm{f}}\right)$ near the top of the alkali metal mixture column in the finger (side-arm). At all times we avoided any mechanical strain and the temperatures were changed very slowly. The experimental setup is shown in Figure 1. 


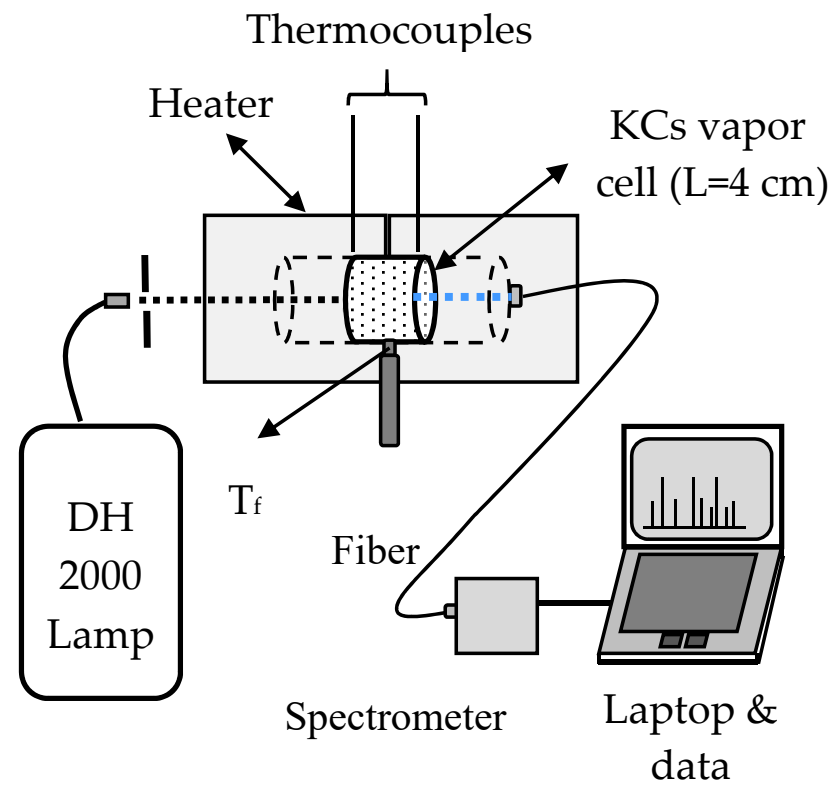

Figure 1. Experimental setup.

A spectrometer in Figure 1 is a special kind of small USB 4000 with enhanced UV option (OceanOptics, ES enhanced sensitivity). It enables large spectral regions to be measured at once for different high temperatures of the alkali mixture and with a low resolution of about $10 \mathrm{~cm}^{-1}$ or more exactly, $0.17 \mathrm{~nm} /$ pixel. From the transmission spectrum, we obtained the spectrum of the absorption coefficient, $\mathrm{k}(\lambda)$, for all temperatures from $\mathrm{T}_{\mathrm{f}}=300^{\circ} \mathrm{C}$ up to $\mathrm{T}_{\mathrm{f}}=600{ }^{\circ} \mathrm{C}$ using Beer-Lambert Law: $\mathrm{k}(\lambda)=1 / \mathrm{L} \ln \left(\mathrm{I}_{\mathrm{o}}(\lambda) / \mathrm{I}(\lambda)\right)$, where $\lambda$ is a wavelength, $\mathrm{L}$ the length of the homogeneous vapor mixture in the cell, and $\mathrm{I}_{\mathrm{o}}(\lambda) / \mathrm{I}(\lambda)$ is the ratio of the intensity at the entrance and the exit from the vapor in the cell.

\section{Results}

In Figure 2a, we present two absorption spectra between 200 and $420 \mathrm{~nm}$ for the two highest $\mathrm{T}_{\mathrm{f}}$ temperatures measured at the top of the vertical side-arm. All measured spectra are from the low-resolution OceanOptics spectrometer. For the UV part of the spectrum, low resolution is justified by the fact that the photoionization of KCs molecule are on one hand subject to ejected electron energy which is not zero but rather spread over a certain energy interval and on the other hand if it is excitation to doubly excited states of $\mathrm{KCs}$ molecule $\left(\mathrm{KCs}^{* *}\right)$, then these states are subject to autoionization. In both cases, UV bands will appear as a structured continuum or quasi-continuum bands. Therefore, the low resolution of the spectrometer does not present experimental inferiority. The resolution of the spectrometer is $700 \mathrm{~nm}$ over 4000 pixels, equal to $0.17 \mathrm{~nm} /$ pixel.

Table 1. Positions of the observed photoionization bands.

\begin{tabular}{ccc}
\hline Band Number & Wavelength (nm) & Wavenumber $\left(\mathbf{c m}^{-\mathbf{1}}\right)$ \\
\hline 1 & 242.7 & 41,203 \\
\hline 2 & 248.8 & 40,192 \\
\hline 3 & $255.8,256[10]$ & 39,093 \\
\hline 4 & 259.1 & 38,595 \\
\hline 5 & 266.4 & 37,537 \\
\hline 6 & 269.3 & 37,133 \\
\hline 7 & $276.3,277[10]$ & 36,192 \\
\hline 8 & 280 & 35,714 \\
\hline
\end{tabular}




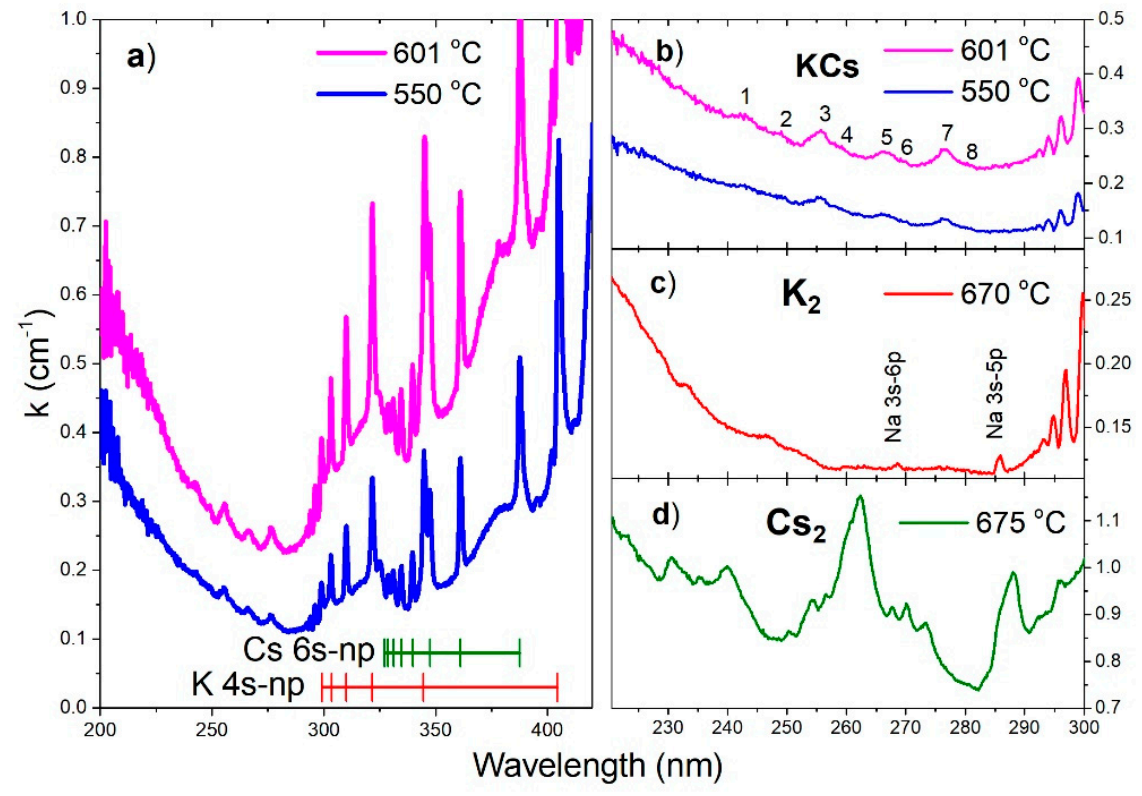

Figure 2. KCs absorption spectra for the two highest temperatures in the 200-420 nm spectral range (Figure 2a). Corresponding background light source was from the deuterium lamp. At the bottom right in Figure 2a, we put connected crosses denoting positions of the K and Cs Principal Series spectral lines from $5 p$ to $10 p$ and from $8 p$ to $15 p$, respectively. An enlarged spectral region, $220-300 \mathrm{~nm}$, is reproduced for $(2 \mathbf{b}) \mathrm{KCs},(2 \mathbf{c}) \mathrm{K}_{2}$, and (2d) $\mathrm{Cs}_{2}$ cases. The (2b) KCs case shows the growing photoionization bands as listed in Table 1 from 1 to $8 . \mathrm{T}_{\mathrm{f}}=601^{\circ} \mathrm{C}$ means the temperature of the finger of the cell.

The main result in Figure 2a,b is the observation of eight photoionization bands as listed in Table 1. Two of these eight bands at 256 and $277 \mathrm{~nm}$ have previously been observed [10]. The band at $266 \mathrm{~nm}$ was not previously observed since it was covered by the presence of the cesium photoionization bands [12]. The cesium contribution in the present experiment is much more suppressed because of the appropriate initial composition of alkali constituents ( $80 \%$ potassium and $20 \%$ cesium). Our previous measurements of the pure potassium vapor did not reveal any significant photoionization bands in the spectral region below $300 \mathrm{~nm}$, at least for measurements at temperatures below $600^{\circ} \mathrm{C}$. The ionization energy of cesium is $31,406.467 \mathrm{~cm}^{-1}$ and the Principal series limit is at $318.84 \mathrm{~nm}$. The ionization energy of potassium is a bit larger $35,009.14 \mathrm{~cm}^{-1}$ and the Principal series limit is at $285.63 \mathrm{~nm}$. In Figure 2a, the grouping of the K and Cs Principal Series lines toward these two limits can readily be seen. The oval shape of the background photoionization continuum stems from overlapping atomic potassium and atomic cesium photoionization processes, both of which are oval. The overlapped atomic photoionization continua possess Seaton-Cooper minimum $[19,20]$. Because of the larger amount of potassium atoms, the Seaton-Cooper minimum in the present case is more determined by the potassium atoms. From Figure $2 \mathrm{~b}$ we see that the minimum of the observed absorption spectrum is located at $285 \mathrm{~nm}$.

\section{Discussion}

The eight photoionization bands of KCs in Table 1 most probably belong to spectral transitions from the ground state of the KCs molecule to excited states of $\mathrm{KCs}^{+}$ion molecule or to doubly excited states of the $\mathrm{KCs}^{* *}$ molecule that lie above the first ionization limit. $\mathrm{KCs}$ is a heteronuclear molecule and there is no restriction due to the gerade-ungerade selection rules. Unfortunately, the relevant potential curves of those doubly excited states (DES) KCs ${ }^{* *}$ (autoionizing states) and the corresponding transition dipole moments are presently not available.

To obtain the cross-section for the photoionization of the KCs molecule, it is necessary to determine the density of KCs molecules at each temperature in an all-sapphire cell, which would be a difficult task. 
Therefore, we concluded that measured absorption coefficients profiles of the bands are sufficiently good information for the existence of the KCs photoionization bands. However, a very rough estimate of the cross-section of the photoionization bands quoted in Table 1 is about $10^{-39} \mathrm{~cm}^{2}$.

\subsection{Direct Photoionization}

There is a direct photoionization process from $\mathrm{KCs}$ molecule to all states of $\mathrm{KCs}^{+}$ion molecule, but it is not a resonance process, because the ejected electron can have an excess of energy:

$$
K C s+U V_{\text {photon }}=K C s^{+}+E_{\text {electron }}
$$

It would be of interest to perform a ZEKE [21] (Zero-Kinetic-Electron-Energy) photoionization experiment which may reveal a possible direct photoionization process. Thus, ZEKE photoionization would reveal peaks in the photoionization experiment which would be correlated with the extrema (mostly minima) in the relevant difference potentials. However, in the present experiment, the amounts of free electron energy given by the excess energy of the absorbed ultraviolet photons will produce certain smearing of the peaks. Besides these minima, the enhancement in the absorption coefficient is caused by the Boltzmann factor at the bottom of $X^{1} \Sigma^{+}(\mathrm{KCs})$, as shown by the broad vertical arrow in Figure 3. The relevant spectral transitions from the bottom of $X^{1} \Sigma^{+}(\mathrm{KCs})$ would be at smaller wavelengths than those quoted in Table 2, as may be concluded by inspecting Figure 3 .

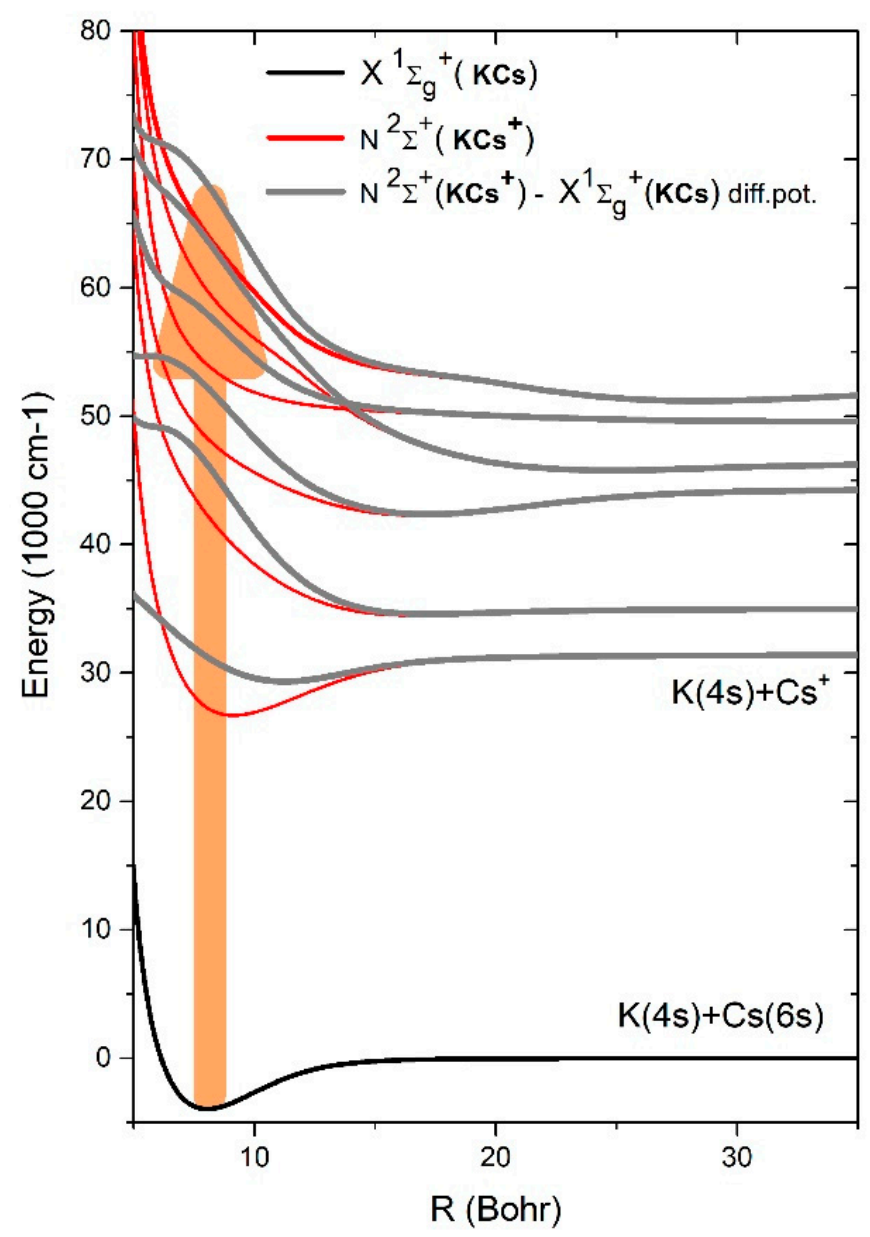

Figure 3. Potential curves of the ground state $\mathrm{KCs}$ molecule with six potential curves of $\mathrm{KCs}^{+}$ molecule $[17,18]$ of ${ }^{2} \Sigma^{+}$symmetry (red lines) together with the relevant difference potentials $N^{2} \Sigma^{+}\left(K C s^{+}\right)-X_{1} \Sigma^{+}(K C s)$ (bold gray lines). 
Table 2. Minima in the difference potentials $N^{2} \Sigma^{+}\left(K C s^{+}\right)-X_{1} \Sigma^{+}(K C s)$.

\begin{tabular}{cccc}
\hline R (bohr) & Difference Energy $\left.\mathbf{( c m}^{-\mathbf{1}}\right)$ & Upper State & Wavelength $(\mathbf{n m})$ \\
\hline 11.34 & 29,338 & $1^{2} \Sigma^{+}$ & 340.9 \\
\hline 17.13 & 34,536 & $2^{2} \Sigma^{+}$ & 289.6 \\
\hline 17.16 & 42,399 & $3^{2} \Sigma^{+}$ & 235.9 \\
\hline 24.90 & 45,785 & $4^{2} \Sigma^{+}$ & 218.4 \\
\hline
\end{tabular}

In Table 2, we give the positions and the energies of the minima in the difference potentials $N^{2} \Sigma^{+}\left(K C s^{+}\right)-X_{1} \Sigma^{+}(K C s)$ that would produce an enhancement in the absorption coefficient if the ejected electron had zero kinetic energy. A comparison of the values of Tables 1 and 2 provide a less satisfactory agreement for the positions of the photoionization bands. We only want to show that such a comparison should be made for all other symmetries of the excited $\mathrm{KCs}^{+}$potential curves (e.g., ${ }^{2} \Pi$, etc.).

\subsection{Indirect Photoionization}

The indirect photoionization has one excitation step before the final ionizations (autoionization). The intermediate step brings the KCs molecule to a doubly excited state (DES) which lies above the first ionization potential. Doubly excited states (DES), $\mathrm{KCs}^{* *}$, have asymptotes lying between $\mathrm{K}(4 \mathrm{~s})+\mathrm{Cs}^{+}$ and $\mathrm{K}(4 \mathrm{p})+\mathrm{Cs}^{+}$asymptotes, pertinent to the present spectral range. The two-step process may be described by the following equations:

$$
\begin{aligned}
& \mathrm{KCs}+U V_{\text {photon }} \rightarrow \mathrm{KCs}^{* *} \\
& \mathrm{KCs}^{* *} \rightarrow \mathrm{KCs}^{+}+E_{\text {electron }} .
\end{aligned}
$$

Here, $\mathrm{KCs}^{* *}$ represents an autoionization state. If it were an atom, instead of a molecule, that transition would be characterized by the Fano profile [14]. However, since we deal with a molecule that rotates and vibrates, the Fano profile will be drastically modified.

It might be worth mentioning that some of the doubly excited molecular states are asymptotically forbidden in a dipole approximation. However, at smaller internuclear separations, those transitions to doubly excited molecular states may be induced by collisions. The KCs* DES are immersed in the ionization continuum of the KCs molecule and are, therefore, subjected to autoionization. All specific spectral transitions will have a certain lifetime connected with the width of the corresponding spectral lines. The value of the autoionization probability of each doubly excited KCs states $\left(\mathrm{KCs}^{* *}\right)$ will, therefore, determine the extent of the smearing of the observed photoionization band shapes. Some of these doubly excited states could be described as stemming from ion-pair states $\mathrm{K}^{+}+\mathrm{Cs}^{*}{ }^{*}$ or $\mathrm{K}^{*-}+\mathrm{Cs}^{+}$, where the negative ions are in the appropriate excited states so that the total energy of the ion-pair molecule is lying above the first ionization limit of the KCs molecule.

In Figure 4, we present the sketch of one possible transition from the neutral KCs ground state to the doubly excited $\mathrm{KCs}^{* *}$ state. In this sketch for the $\mathrm{KCs}^{* *}$ state, we took the $\mathrm{KCs}^{+}$ground state potential from the $K(4 s)+C s^{+}$asymptote and shifted vertically towards the $K^{*}(4 p)+C s^{*}(8 s)$ asymptote. This artificial potential can be used to construct the relevant difference potential, which in turn probably has a minimum which would produce an enhancement in the absorption coefficient (usually a satellite band in the quasi-static wing of spectral lines [22]). This enhanced band would be identified as one peak among the observed photoionization band (Table 1). 


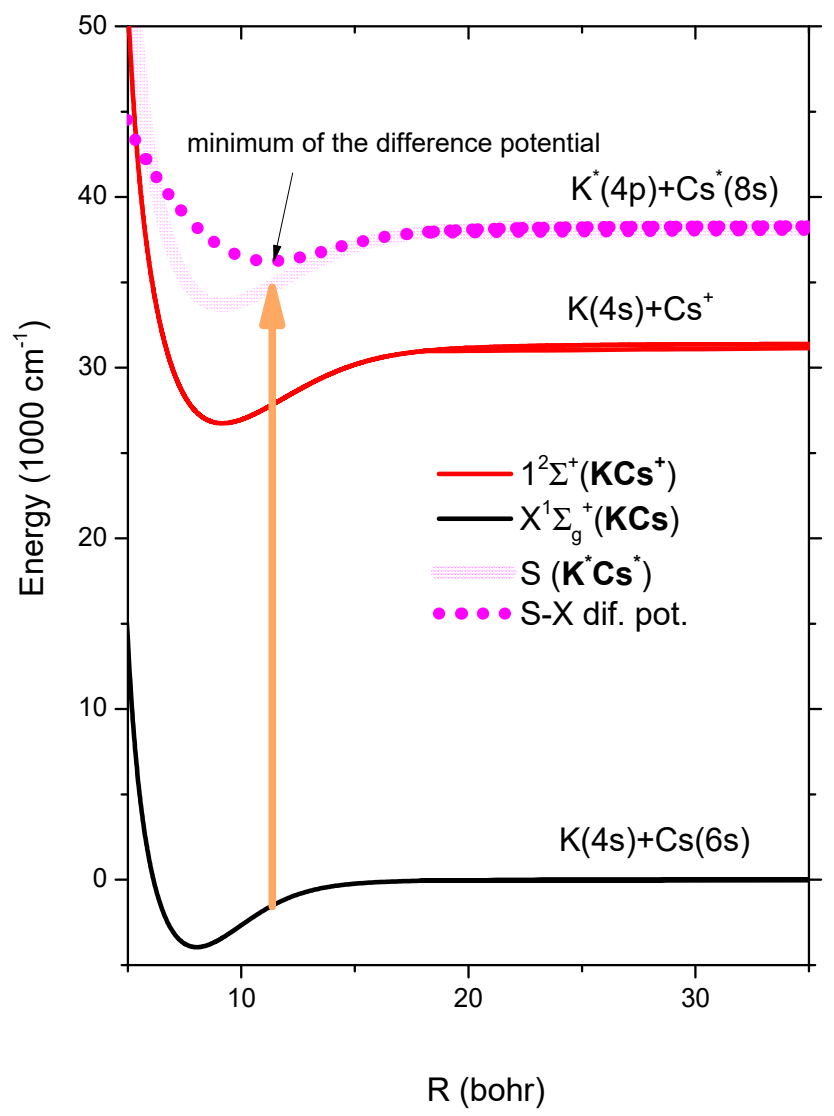

Figure 4. The ground state of the $\mathrm{KCs}^{+}$molecule (red) and the sketch of an artificially constructed, doubly excited state (DES of $\mathrm{KCs}^{* *}$ denoted by S) with the asymptote $\mathrm{K}^{*}(4 \mathrm{p})+\mathrm{Cs}^{*}(8 \mathrm{~s})$, lying above the lowest $\mathrm{KCs}^{+}$potential curve. The minimum of the relevant difference potential of the transition $\mathrm{S}\left(\mathrm{KCs}^{* *}\right)-\mathrm{X}(\mathrm{KCs})$ (magenta color) points to the location of the one possible photoionization band.

Doubly excited states of diatomic molecules that lie above the first ionization limit are well known in the case of the $\mathrm{H}_{2}$ molecule $[23,24]$. However, those states are so high that the homologous transitions to those as discussed in the present paper would lie in the VUV spectral region. As discussed in [25], both the above discussed photoionization processes end up in the same state, $\mathrm{KCs}^{+}+E_{\text {electron }}$. This means that we have the interference between these two pathways, and this interference produces a special structure that we observe as a structured continuum that has band peaks, as quoted in Table 1 .

\section{Materials and Methods}

We produced very high densities of potassium-cesium vapor mixture in a specially designed, all-sapphire cell. This enabled the creation of a well-defined column of KCs vapor which allowed the determination of the absorption coefficient in the spectral region between 220 and $300 \mathrm{~nm}$. In this wavelength interval, a few photoionization bands appeared and denoted by the maximum of the profile. Another two all-sapphire cells with pure potassium and pure cesium were used in a similar experimental condition for comparison to extract the photoionization bands of KCs molecule. The interpretation of the observed bands was discussed by the direct and indirect photoionization process. We also mentioned a possible interference of these direct and indirect photoionization transitions as a process behind the formation of the KCs photoionization bands.

\section{Conclusions}

Experimental observation of the eight photoionization bands of the KCs molecule in the deep ultraviolet spectral region presents a challenge for the future quantum-mechanical calculations. 
At present, we tried to test the idea that some of the observed photoionization bands can be interpreted as stemming from the direct photoionization transition from the neutral KCs molecule to the ion $\mathrm{KCs}^{+}$molecule. We did not find more than just a few possible coincidences for such transitions and, therefore, concluded that the KCs photoionization bands stem from the one-photon transitions to doubly excited states of the KCs molecule lying above the first ionization potential. Unfortunately, such doubly excited KCs states have not yet been theoretically treated. However, both direct and indirect photoionization processes may interfere enabling the creation of the observed photoionization bands. This will be the main point in our future investigations.

Author Contributions: G.P. discussed the problem, made the experiment, prepared the figures, wrote the paper; R.B. discussed the problem, wrote the paper; J.K. discussed the problem, D.S. discussed the problem, wrote the paper; N.J. and J.M. made the experiment. All authors have read and agreed to the published version of the manuscript.

Funding: This research received no external funding.

Acknowledgments: The support by General Facility projects GS03/01 and GS01/08 of Kuwait University Research sector is gratefully acknowledged. The theoretical part of the present work was supported by the Croatian Science Foundation (HRZZ) under project number 2753.

Conflicts of Interest: The authors declare no conflict of interest.

\section{References}

1. Rakić, M.; Beuc, R.; Bouloufa-Maafa, N.; Dulieu, O.; Vexiau, R.; Pichler, G.; Skenderović, H. Satellite bands of the RbCs molecule in the range of highly excited states. J. Chem. Phys. 2016, 144, 204310. [CrossRef]

2. Szczepkowski, J.; Grochola, A.; Kowalczyk, P.; Jastrzebski, W. Spectroscopic study of the $\mathrm{C}(3) 1 \Sigma+\leftarrow \mathrm{X} 1 \Sigma+$ and $\mathrm{c}(2) 3 \Sigma+\leftarrow \mathrm{X} 1 \Sigma+$ transitions in KCs molecule. J. Quant. Spectrosc. Radiat. Transfer. 2018, 204, 131-137. [CrossRef]

3. Birzniece, I.; Nikolayeva, O.; Tamanis, M.; Ferber, R. Potential construction of the B(1)1П state in KCs based on Fourier-Transform spectroscopy. J. Quant. Spectrosc. Radiat. Transfer. 2015, 151, 1-4. [CrossRef]

4. Busevica, L.; Klincare, I.; Nikolayeva, O.; Tamanis, M.; Ferber, R.; Meshkov, V.; Pazyuk, E.A.; Stolyarov, A.V. Fourier transform spectroscopy and direct potential fit of a shelflike state: Application to E (4) $1 \Sigma+\mathrm{KCs}$. J. Chem. Phys. 2011, 134, 104307. [CrossRef] [PubMed]

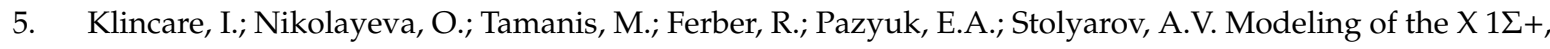
a $3 \Sigma+\rightarrow \mathrm{E}(4) 1 \Sigma+\rightarrow \mathrm{X} 1 \Sigma+(\mathrm{v}=0, \mathrm{~J}=0)$ optical cycle for ultracold KCs molecule production. Phys. Rev. A 2012, 85, 062520. [CrossRef]

6. Borsalino, D.; Vexiau, R.; Cachan, M.; Luc-Koenig, E.; Dulieu, O.; Bouloufa-Maafa, N. Prospects for the formation of ultracold polar ground state KCs molecules via an optical process. J. Phys. B At. Mol. Opt. Phys. 2016, 49, 055301. [CrossRef]

7. Creek, D.M.; Marr, G.V. Some ultraviolet cross-section measurements on molecular alkali-metal vapors. J. Quant. Spetrosc. Radiat. Transfer. 1968, 8, 1431. [CrossRef]

8. Hotop, R.; Niemax, K. Continuum- and bound-state pair absorption in Cs-Cs, Rb-Rb and Cs-Rb systems. J. Phys. B At. Mol. Phys. 1980, 13, L93-L99. [CrossRef]

9. Hotop, R.; Niemax, K.; Schlüter, D. On pair-absorption in intrinsic vapors. Z. Phys. A Atoms. Nucl. 1982, 304, 185. [CrossRef]

10. Hotop, R.; Niemax, K.; Richter, J.; Weber, K.-H. Thermionic detection of the ionic fragments of continuum-state pair absorption systems. Opt. Commun. 1981, 36, 35-38. [CrossRef]

11. Suemitsu, H.; Samson, J.A.R. Relative photoionization cross sections of Cs, Cs2, Rb, and Rb2. Phys. Rev. A 1983, 28, 2752-2758. [CrossRef]

12. Pichler, G.; Makdisi, Y.; Kokaj, J.; Thomas, N.; Mathew, J. Structured photoionization continuum of superheated cesium vapor. J. Phys. B At. Mol. Opt. Phys. 2015, 48, 165002. [CrossRef]

13. Rakić, M.; Pichler, G. Photoionization bands of rubidium molecule. J. Quant. Spectrosc. Radiat. Transfer. 2018, 208, 39. [CrossRef]

14. Fano, U. Effects of Configuration Interaction on Intensities and Phase Shifts. Phys. Rev. 1961, 124, 1866-1878. [CrossRef] 
15. Beuc, R.; Movre, M.; Horvatić, B.; Čopor, M.; Vdović, S.; Nevsesyan, A.; Varzhapetyan, T.; Sarkisyan, D.; Pichler, G. RbCs bands observation and interpretation. Appl. Phys. B 2007, 88, 111-115. [CrossRef]

16. Allouche, A.-R.; Korek, M.; Fakherddin, K.; Chaalan, A.; Dagher, M.; Taher, F.; Aubert-Frecon, M. Theoretical electronic structure of RbCs revisited. J. Phys. B At. Mol. Opt. Phys. 2000, 33, 2307-2316. [CrossRef]

17. Korek, M.; Allouche, A.R.; Fakhreddine, K.; Chaalan, A. Theoretical study of the electronic structure of LiCs, NaCs, and KCs molecules. Can. J. Phys. 2000, 78, 977. [CrossRef]

18. Korek, M.; Allouche, A.R.; Abdul, S.N. Al Potential curves and rovibrational energies for electronic states of the molecular ion KCs+. Can. J. Phys. 2002, 80, 1025-1035. [CrossRef]

19. Seaton, M.J. A comparison of theory and experiment for photo-ionization cross-sections II. Sodium and the alkali metals. Proc. R. Soc. A 1951, 208, 418.

20. Cooper, J.W. Photoionization from Outer Atomic Subshells. A Model Study. Phys. Rev. 1962, 128, 681-693. [CrossRef]

21. Müller-Dethlefs, K.; Schlag, E.W. High-resolution zero kinetic energy (ZEKE) photoelectron spectroscopy of molecular systems. Annu. Rev. Phys. Chem. 1991, 42, 109-136. [CrossRef]

22. Movre, M.; Pichler, G. Resonance interaction and self-broadening of alkali resonance lines I. Adiabatic potential curves. J. Phys. B At. Mol. Phys. 1977, 10, 2631. [CrossRef]

23. Sanchez, I.; Martin, F. The doubly excited states of H2 molecule. J. Chem. Phys. 1997, 106, 77. [CrossRef]

24. Sanchez, I.; Martin, F. Phys Origin of Unidentified Structures in Resonant Dissociative Photoionization of H2. Rev. Lett. 1997, 79, 1654. [CrossRef]

25. Palacios, A.; Feist, J.; Castrillo, A.G.; Sanz-Vicario, J.L.; Martín, F. Autoionization of Molecular Hydrogen: Where do the Fano Lineshapes Go? ChemPhysChem 2013, 14, 1456-1463. [CrossRef] [PubMed]

(C) 2020 by the authors. Licensee MDPI, Basel, Switzerland. This article is an open access article distributed under the terms and conditions of the Creative Commons Attribution (CC BY) license (http://creativecommons.org/licenses/by/4.0/). 